\title{
El principio de la integralidad en la formación de personal aeronáutico y gestión del riesgo.
}

\author{
Rubén Darío Lésmes Bustamante
}

Piloto -Instructor de Vuelo -Conferencista -Consultor aeronáutico

Junio 2021

\section{Resumen:}

La inclusión de las habilidades no técnicas en el perfil del individuo que se desempeña en el sector aeronáutico es sin duda alguna uno de los aspectos que más ha generado resultados en la mitigación del riesgo, hemos avanzado en la comprensión de las competencias humanas requeridas para el comportamiento del individuo considerando a las siguientes: Las competencias técnicas, las cognitivas y ahora las actitudinales.

El objetivo de la presente reflexión es incentivar y motivar a las organizaciones del sistema aeronáutico para que se incluyan a las competencias no técnicas en los aspectos clave de la administración del recurso humano, para esto, El principio de la integralidad contiene la integración de manera articulada de éstas competencias no técnicas en los procesos de selección, formación, promoción y evaluación de los individuos que pertenecen al sistema aeronáutico.

Se ha evidenciado que los procesos de formación básica de personal aeronáutico aún no incluyen a las competencias no técnicas como una herramienta clave del proceso, incluso los programas especiales de gestión del riesgo se han promovido de manera técnica y cognitiva dejando un vacío en la estructura del comportamiento, esto afecta indudablemente el desempeño cuando el personal enfrenta situaciones no deseadas o de riesgo.

Como conclusión del presente estudio se ha determinado que sin lugar a duda es necesario desarrollar un plan de actualización de los procesos de la administración del factor humano en donde se incluya la integralidad de las competencias como estrategia clave en la adherencia de la cultura de la seguridad operacional y en el proceso de gestión del riesgo aeronáutico.

La participación sistémica de las organizaciones aeronáuticas en este emprendimiento se hace relevante si consideramos que los comportamientos de los individuos afectan el cumplimiento de los objetivos comunes en materia de la seguridad operacional.

Debe impulsarse además la participación del profesional en Psicología aeronáutica en el proceso de formación del personal. 


\title{
El principio de la integralidad en la formación de personal aeronáutico y gestión del
} riesgo.

\author{
(Artículo de reflexión)
}

El documento 9859 de la OACI define a la seguridad operacional como: "Estado en que el riesgo de lesiones a las personas o daños a los bienes se reduce y se mantiene en un nivel aceptable o por debajo del mismo, por medio de un proceso continuo de identificación de peligros y gestión de riesgos." (1). Disminuir o mitigar el riesgo de lesiones a las personas y daños a los bienes hasta un nivel aceptable se logra (de acuerdo a la definición) mediante dos actividades o acciones: Identificar peligros y gestionar el riesgo, estas actividades aparentemente sencillas plantean un reto enorme, promover las acciones del personal de las organizaciones a todo nivel en este sentido, no obstante, las acciones del personal están fuertemente afectadas por la naturaleza de su cultura.

La cultura de un individuo está compuesta por varias sub culturas como lo son entre otras: La cultura geográfica, la cultura de género, la cultura generacional, la cultura organizacional, la cultura profesional y otras más que han inculcado arraigos en el comportamiento. Si se pretende inducir comportamientos comunes (identificar peligros y gestionar el riesgo) en las personas que se desempeñan en el sistema aeronáutico se hace necesaria la inclusión de una nueva cultura que identifique a estas personas a través de características actitudinales comunes, ésta cultura es la llamada cultura de la seguridad operacional.

\section{La cultura de la seguridad operacional}

La percepción que tienen los individuos sobre los aspectos del riesgo difieren de unos a otros según sea su arraigo cultural o su vivencia particular, en el artículo "La conducta humana frente a los riesgos laborales: determinantes individuales y grupales" (2), publicado por Esther Puyal Español, en la revista: Acciones e investigaciones sociales, número 12 de 2001 se describe la percepción del riesgo según estos arraigos:

"La psicosociología ha concedido una gran importancia al estudio de los procesos socio cognitivos por sus implicaciones en la conducta, uno de los principios básicos de este campo es que las personas al observar la realidad no lo hacen de una manera neutral, sino que sus necesidades, deseos, expectativas, valores, aprendizajes o conocimientos previos influyen en lo que ven y recuerdan. Es en estos factores de carácter psicosocial donde se ha hecho mayor hincapié en los trabajos sobre cogniciones; si bien pueden identificarse otros factores de carácter 
situacional que también influyen en la percepción. En este sentido, diríamos que es el conjunto total de fuerzas que interactúan en un momento determinado o «campo de fuerzas» el que condicionará el proceso perceptivo de modo que, una situación puede ser percibida como amenazante por una persona, y a otra resultarle estimulante, o incluso una misma persona puede percibirla de distinto modo en dos momentos distintos. la percepción no es un proceso objetivo, sino un proceso selectivo, dinámico y funcional. Un proceso determinado fuertemente por las características permanentes y temporales de las personas."

Esta percepción particular de los individuos afecta sin lugar a duda la gestión del riesgo de manera organizacional, por lo tanto, el sistema aeronáutico planteó la necesidad de incluir mediante una cultura a todas aquellas características que faciliten el actuar de manera colectiva.

De acuerdo al documento 9859 de la OACI parte 2.8 (3), "La cultura puede describirse en los términos más sencillos como "programación colectiva de la mente". Una de las descripciones más gráficas de la cultura la presenta como soporte lógico de la mente. La cultura influye en los valores, las creencias y los comportamientos que compartimos con otros miembros de los diversos grupos sociales a que pertenecemos. La cultura sirve para vincularnos como miembros de grupos y proporciona claves sobre la forma de comportarse tanto en situaciones normales como inhabituales. La cultura establece las reglas del juego, o el marco para todas nuestras interacciones interpersonales. Es la suma total de la forma en que las personas llevan adelante sus asuntos en un medio social particular y proporciona un contexto en que las cosas ocurren. En términos de gestión de la seguridad operacional, comprender la cultura es tan importante como comprender el contexto, dado que la cultura es un determinante importante de la actuación humana."

La adherencia de los comportamientos en materia de gestión, se logra mediante la asimilación de las características actitudinales comprendidas en la cultura de la seguridad operacional; la información y la socialización de éstas características no son suficientes para lograr el objetivo propuesto en el direccionamiento organizacional de la actuación humana.

Se requiere una estrategia adecuada de promoción que incentive el actuar de los individuos más allá de la comprensión de contenidos, el Boletín de la Organización de Aviación Civil Internacional, volumen 61, número 6 en su artículo sobre: Los sistemas de gestión de la seguridad ATM son una prioridad para Europa, (4) menciona que "La promoción generalizada de los principios de gestión de la seguridad operacional es esencial para los esfuerzos europeos de mejorar la seguridad."

La promoción de la seguridad operacional es un pilar fundamental en la estructura del SMS (Safety Management System) por lo que se ha reglamentado su inclusión a nivel organizacional, el Reglamento Aeronáutico de Colombia parte 219 es un ejemplo de ello cuando menciona en su aparte 219.105 (5), Estructura de un SMS, literales (d), (1), (i) " $E l$ proveedor de servicios creará y mantendrá un programa de instrucción en seguridad 
operacional que garantice que el personal cuente con la instrucción y las competencias necesarias para cumplir sus funciones en el marco del SMS".

La asimilación de la seguridad operacional como una cultura no se da por la promoción de las competencias técnicas o cognitivas de los individuos, las competencias a las que se refiere el reglamento son fundamentalmente competencias actitudinales. El uso de competencias no técnicas como: Toma de decisiones, liderazgo, resiliencia, conciencia situacional, manejo del entorno, comunicación asertiva, entre otras, es lo que finalmente ayuda en la adherencia del comportamiento.

La cultura de la seguridad operacional como el conjunto de características comunes que identifican el actuar del personal aeronáutico se adapta y se adhiere a las organizaciones del sistema a todo nivel y debe permear al total de los individuos que interactúan en la actividad de la gestión del riesgo como una filosofía de estandarización.

El comportamiento basado en estándares y en competencias técnicas y no técnicas, marca la diferencia en cuanto a la mitigación de los riesgos a los que está expuesta la actividad del sector, integrar las competencias de manera articulada como parte de las campañas de promoción de la seguridad operacional mejora radicalmente la asimilación de la misma.

\section{Factores determinantes en el estudio de la accidentalidad y las competencias}

La disminución de la probabilidad y la severidad de los eventos no es una gestión nueva, éste objetivo ha sido planteado desde el génesis de la aviación. El reto es trasladar un aparato aéreo del punto $A$ al punto $B$, no obstante, éste traslado no puede ejecutarse de manera improvisada ya que en definitiva el traslado debe contener la condición de seguridad, así entonces, trasladar un aparato aéreo del punto A al punto B de manera segura ha sido consecuencia de múltiples esfuerzos y estudios contenidos en la evolución misma de la industria aeronáutica.

Se pueden identificar tres eras o etapas en las que la estrategia de gestión del riesgo ha cambiado considerablemente dependiendo de los factores que han sido considerados como riesgos o amenazas en el sector. Según Andrew R. Hale \& Jan Jovden en el texto Ocupational injury, risk prevention and intervention, capítulo 11, Management and culture, the third age of safety, a review of approaches to organizational aspects of safety, healt and enviroment. 1998, (6), éstos factores contribuyentes han sido definidos como: Factores técnicos, factores humanos y factores organizacionales o seguridad operacional. También la OACI (Organización de Aviación Civil Internacional) en su documento 9859 Manual de gestión de la seguridad operacional, capítulo 2. Conceptos básicos de seguridad operacional, muestra los factores que han influenciado el pensamiento en materia de la seguridad operacional. (ver gráfica 1.0). 


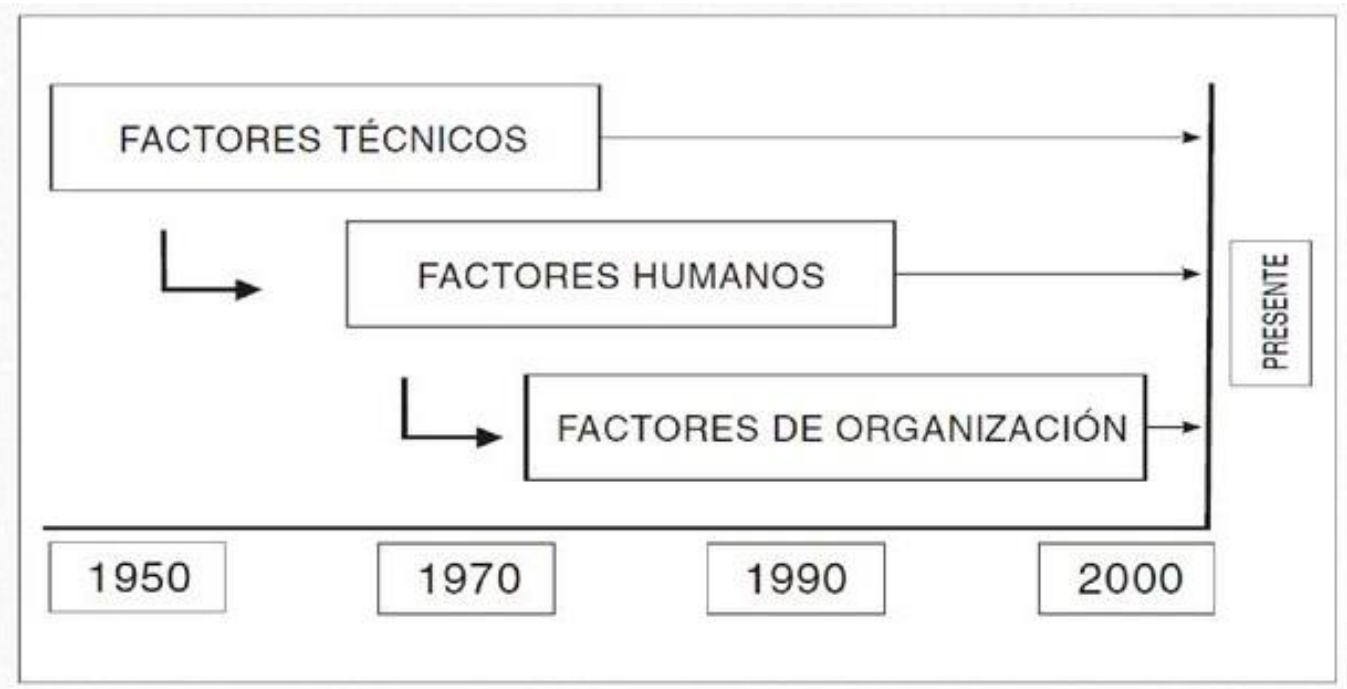

Grafica 1.0

Evolución del pensamiento en materia de seguridad operacional

OACI - Doc. 9859 Manual de gestión de la seguridad operacional

Capítulo 2. Conceptos básicos de seguridad operacional.

La evolución del pensamiento en materia de la gestión se fundamenta principalmente en que, en el inicio del vuelo de los aerodinos, años 1900's se consideró que hacer más segura a la actividad aérea se fundamentaba en el mejoramiento del componente técnico de la industria, esto es: Materiales de construcción, sistemas de navegación, sistemas de control de tránsito, análisis meteorológico, etc. Es apenas comprensible que en el inicio de la actividad aérea la gran mayoría de accidentes tenían como causa probable el uso de materiales y sistemas inadecuados o inexistentes.

La evolución tecnológica continúa aún hoy en día, incluso cuando ya contamos con sistemas y componentes de altísima confiabilidad y precisión.

En los años 50's se pudo evidenciar que, aunque ya se contaba con aparatos aéreos tecnificados y sistemas de advertencia, control y navegación de precisión aceptable, los índices de la accidentalidad no disminuyeron según la expectativa de la industria, es allí en donde inicia la segunda era o etapa de gestión que se fundamentó en el factor humano. La OACI presentó el documento 9683 “Manual de. Instrucción sobre Factores Humanos," (7), éste es un acercamiento a la comprensión de las necesidades de entrenamiento del personal en lo relacionado a la medicina de aviación y a la psicología aeronáutica, es importante mencionar que en este documento no se proyecta un estudio detallado de las competencias no técnicas de los individuos, sino que, más bien se proyecta a la psicología desde un aspecto clínico, quizás el avance más significativo en aras de las competencias 
no técnicas contenido en este documento sea el desarrollo en los años 70's del programa CRM (Crew Resource Management), que involucra aspectos de trabajo en equipo y administración de cargas laborales abordo.

Muy a pesar de los avances en el estudio del factor humano y su desempeño basado fundamentalmente en lo clínico y el CRM, la segunda era de gestión no trajo consigo cambios significativos en la tendencia de la accidentalidad. Es a partir de los años 90’s cuando surge entonces la tercera era o etapa de gestión llamada la era de los factores organizacionales. En esta era, se evidencia que el comportamiento del personal operativo tanto de mantenimiento como de despacho, auxiliares de vuelo y pilotos, es afectado por los procesos organizacionales y éstos a su vez son consecuencia de las políticas institucionales, que se transformaron con el tiempo en los procesos organizacionales.

Es importante decir que el hecho de evolucionar con las eras, no determina que cada una de ellas haya dejado de ser una estrategia de gestión, por lo contrario, la gestión técnica, la de factores humanos y la de aspectos organizacionales se integran unas con otras en el actual sistema aeronáutico.

Entender la evolución del pensamiento en materia de la gestión de la seguridad cobra importancia si se relaciona transversalmente con el enfoque que se ha dado a las competencias requeridas por el personal según cada era de manera específica.

Existe una correlación entre las necesidades de gestión y las competencias requeridas según la necesidad de cada era así:

1. La era de los factores técnicos que se fundamentaba en la necesidad de mejorar los materiales y sistemas de control y vigilancia requerían de los individuos competencias técnicas basadas en habilidad.

2. Los años posteriores a la década de los 50's se contaba con sistemas y tecnologías en las aeronaves que requerían el conocimiento de los mismos por parte de quienes los operaban y reparaban e incluso de todos aquellos quienes participaban en la operación aérea, era necesario el estudio y conocimiento de las tecnologías, cartografía, sistemas, procedimientos, etc. Las competencias técnicas basadas en la habilidad dieron paso a las competencias técnicas basadas en el conocimiento o a las que llamamos habilidades cognitivas (las habilidades de coordinación, orientación y control de vuelo cedieron en gran parte debido a la operación de pilotos automáticos, sistemas de navegación y control de vuelo autónomo).

3. La gestión basada en factores organizacionales que se desarrolló como una cultura organizacional y que en ultimas es la cultura de la seguridad operacional, se fundamenta en la capacidad de actuar conforme a lo establecido o a lo estandarizado, recordemos que la cultura organizacional y por ende la de la seguridad operacional busca unificar el comportamiento en materia de la gestión a todo nivel organizacional en las actividades de mitigación del riesgo. El 
comportamiento se fundamenta en la nuevas competencias no técnicas o actitudinales.

Al estudiar la tendencia de la probabilidad de los eventos en seguridad según las estadísticas presentadas por Aviation Safety Network, (ver gráfica 2.0)

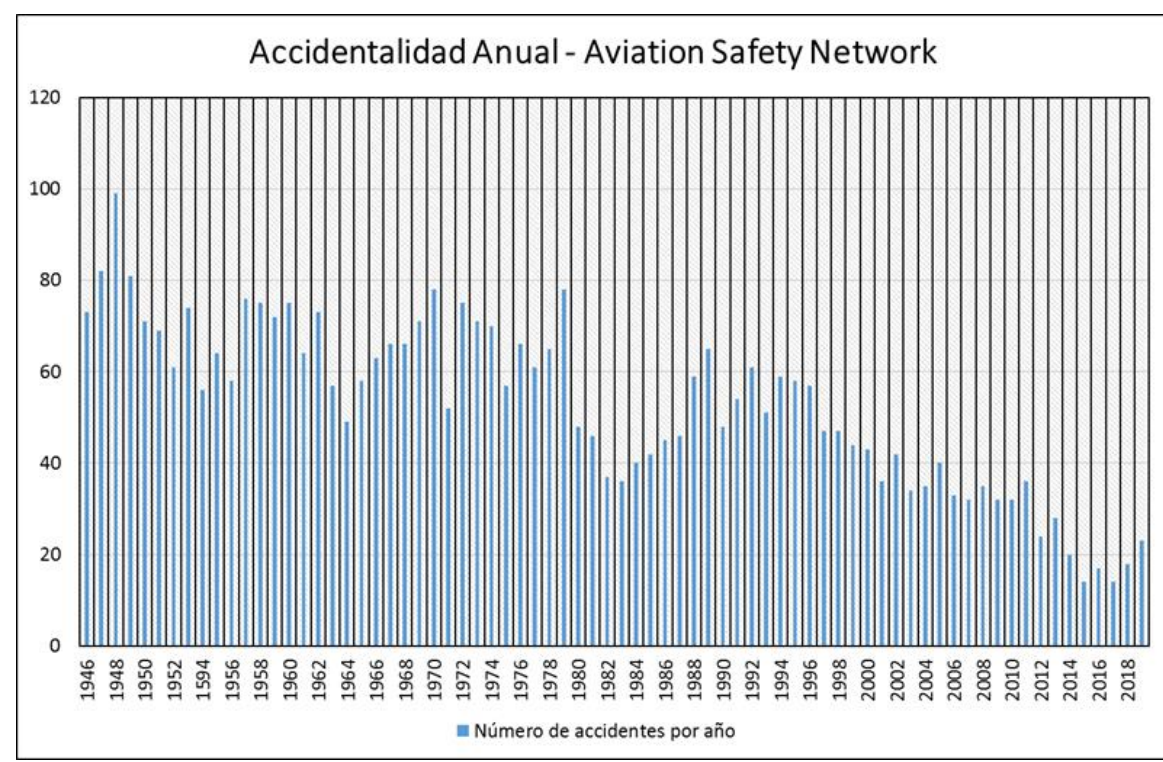

Gráfica 2.0

https://aviation-safety.net/statistics/

Fatal Accidents Per Year 1946-2019

se puede determinar que es a partir de la década de los 90's durante el desarrollo e implementación de la seguridad operacional y el SMS, cuando la tendencia de la accidentalidad disminuye casi en una gradiente constante.

La correlación entre la estrategia de gestión organizacional y los índices de la accidentalidad es relevante y concluyente, la inclusión de los procesos organizacionales en conjunto con las competencias no técnicas o actitudinales han logrado la mitigación del riesgo aeronáutico.

Flight Safety Foundation ha publicado mediante su biblioteca Skybrary un artículo relacionado al programa EBT (Evidence Based Training), (8), en él se describe con claridad la inclusión de las competencias no técnicas como determinantes en la gestión de la seguridad.

"EBT recognises the need to develop and evaluate crew performance according to a set of competencies without necessarily distinguishing between the 'non-technical' (e.g. CRM) and 
the 'technical' competencies needed in order to operate safely. Any area of competence assessed by EBT instructors not to meet the required level of performance needs to be associated with an observable behaviour that could lead to an unacceptable reduction in safety margins.

Under EBT the following are the competencies that trainees are required to demonstrate and EBT instructor are required to be able to accurately assess: 1. Application of Procedures; 2. Communication; 3. Aircraft Flight Path Management, automation; 4. Aircraft Flight Path Management, manual control; 5. Leadership and Teamwork; 6. Problem Solving and Decision Making; 7. Situation Awareness; 8. Workload Management

"EBT reconoce la necesidad de desarrollar y evaluar el rendimiento de las tripulaciones de acuerdo a un set de competencias que no necesariamente difieren entre las no técnicas (e.g. CRM) y las competencias técnicas necesarias para operar de manera segura. Cualquier área de competencia juzgada por los instructores EBT que no reúna el nivel adecuado de rendimiento necesario asociado a un comportamiento observable podría direccionar una reducción inaceptable de los márgenes de seguridad.

Bajo el EBT las siguientes son las competencias que los entrenados deben demostrar y el instructor EBT está llamado requerir para poder juzgar de manera precisa: 1. Aplicación de procedimientos; 2. Comunicación; 3. Administración de la senda de vuelo del avión automatización; 4. Control manual de la senda de vuelo del avión; 5. Liderazgo y trabajo en equipo; 6. Resolución de problemas y toma de decisiones; 7. Conciencia situacional; 8. Asignación y distribución del trabajo".

La inclusión de las competencias no técnicas en la gestión del riesgo no desconoce la necesidad de las competencias técnicas y cognitivas de los individuos, más bien se integran en el proceso organizacional; se hace necesario una política o compromiso en las organizaciones del sistema aeronáutico para con ésta integración, allí cobra especial relevancia El principio de la integralidad, publicado por el autor en octubre de 2020, (9).

\section{Programas especiales de gestión del riesgo}

A partir de la comprensión de la necesidad de llevar la gestión del riesgo aeronáutico a todos los niveles organizacionales y una vez estudiado el factor humano más allá de lo clínico, la industria ha generado programas y modelos que orientan la estrategia de mitigación basada en evidencias, la evidencia se obtiene a través de la medición de los factores contribuyentes a la accidentalidad, la casuística en sí misma es la materia prima para la elaboración de estos programas y modelos.

Podemos enunciar entre otros los siguientes programas y modelos: Modelo TEM (Threat and Error Management), Programa CFIT (Controlled Flight in to Terrain), Programa ALARP (Approach and Landing Accident Reduction program), LOFT (Line Oriented Flight Training), EBT (Evidence Based Training). 
Lo relevante al estudiar todos y cada uno de los programas y modelos de gestión del riesgo es que en cada uno de ellos identifica al componente actitudinal como el diferenciador entre una condición segura o una condición no deseada, como ejemplo podemos ver en el modelo TEM, cómo, diferentes agencias internacionales lo incluyen con el objetivo claro de moldear comportamientos y actitudes de las tripulaciones de vuelo. Presentamos como ejemplo EASA (10). "EASA a través de la Parte FCL2 y la Organización de Aviación Civil Internacional (ICAO) exigen que se introduzcan los factores humanos y la TEM en todas las formaciones de pilotos en cada fase de vuelo, todos los pilotos, desde los alumnos hasta los profesionales, deben demostrar 'actitudes y comportamientos adecuados para realizar de forma segura un vuelo, incluyendo el reconocimiento y la gestión de posibles amenazas y errores."

Los programas especiales de gestión del riesgo promueven las competencias no técnicas según sea el caso, en el programa ALARP se promueven las competencias en la toma de decisiones y comunicación entre otras, en el programa CFIT, se resalta la conciencia situacional y la complacencia como factores determinantes de gestión, en el EBT se identifican las competencias no técnicas de una manera detallada. (11) "Building a new competency-based training philosophy".

"The ICAO Evidence-Based Training (EBT) concept is a safety-improvement initiative part of the ITQI project (IATA Training and Qualification Initiative) that precisely addresses this challenge by prioritizing the development and assessment of a finite number of key competencies. The EBT basically recommends to train competencies (not events) and choose training scenarios based on evidence collected from in service data to make sure pilots are able to demonstrate a good performance in front of realistic threats and errors."

"El concepto OACI de entrenamiento basado en evidencia (EBT) es una parte de la iniciativa del proyecto ITQI (IATA Training and Qualification Initiative) para la implementación de seguridad operacional, que precisamente direcciona sus esfuerzos priorizando el desarrollo y aseguramiento de un número finito de competencias clave. El EBT básicamente recomienda entrenar competencias (no eventos) y escoger escenarios de entrenamiento basados en evidencias recolectadas de información en servicio para hacer pilotos capaces y demostrar un buen rendimiento en caso de enfrentar amenazas y errores."

Se hace visible en la estructura de los programas especiales de gestión del riesgo que, aunque éstos tienen como consecuencia final un mejor desempeño de las tripulaciones en el control de las aeronaves y en la ejecución de procedimientos abordo, son las competencias no técnicas las que finalmente logran el cumplimiento de los objetivos propuestos en la mitigación de los riesgos, sea que se logre mediante la resiliencia cuando las tripulaciones se enfrentan a situaciones no esperadas o cuando se hace importante la toma de decisiones, la comunicación asertiva, el trabajo en equipo, el liderazgo, etc. Ante las amenazas o errores.

Las tripulaciones de vuelo que participan en el sistema aeronáutico actuando desde sus organizaciones, así como las entidades que participan en la operación aérea, deben 
asimilar y adherir políticas y compromisos claros en el entrenamiento y aseguramiento de las competencias técnicas y no técnicas de manera integrada, éstas políticas están alineadas con la implementación de la cultura de la seguridad operacional.

\section{Administración del recurso humano en la aviación}

Cuando hablamos de la administración del recurso humano en la aviación nos referimos los procesos y a la forma en que los integrantes de las organizaciones a todo nivel son

seleccionados, entrenados, promovidos, y evaluados; ésta administración se ejecuta basada en las competencias requeridas para el desempeño de las funciones según sea la responsabilidad adquirida dentro del sistema aeronáutico, así entonces, la administración del recurso humano difiere en cierto grado según la cultura organizacional de la industria, en teoría, debieran existir diferencias significativas en la administración de pilotos, técnicos, despachadores, controladores, etc.

En las conclusiones del artículo "La cultura organizacional, su importancia en el desarrollo de las empresas" publicado por la universidad internacional del Ecuador en la revista INNOVA RESEARCH JOURNAL (12), se lee lo siguiente:

"Se puede asegurar que las culturas generaran compromiso con los valores corporativos o la filosofía de gestión, lo que permitiría que los empleados trabajen por algo en lo que creen. Las culturas sirven como un sistema de control en la organización, debido a que de ellas derivan los procesos, reglas y formas que generan los patrones de comportamiento, finalmente existen estudios que aseguran que algunos tipos de culturas organizacionales están asociadas con una mayor productividad y aparentemente, las corporaciones bien administradas del mundo tienen culturas distintivas que son de alguna manera responsables de su capacidad de crear, implementar y mantener sus posiciones de liderazgo mundial

La cultura organizacional fragua el comportamiento de los empleados de las instituciones y debido a esto afecta el desempeño empresarial. La cultura organizacional tiene un poderoso efecto en el desempeño y eficacia a largo plazo de las organizaciones. La investigación empírica ha producido una serie impresionante de hallazgos que demuestran la importancia de la cultura a la mejora del desempeño organizacional"

A pesar de la existencia de diversas culturas organizacionales dentro de una empresa aeronáutica, desde la década de los años 90's (cuando la gestión del riesgo migra a los factores organizacionales) se hace evidente que existen elementos o factores comunes en los requerimientos de las competencias actitudinales y en la administración de los individuos que conforman las empresas del sector. 
Un ejemplo claro de esta unicidad encontrada por el sistema aeronáutico, puede entenderse en la descripción y el concepto del Accidente institucional, en él, se describen cinco correlaciones al interior de las organizaciones así: 1. Las condiciones del lugar de trabajo, 2. Las averías activas, 3. Las condiciones latentes, 4. Las defensas y 5. Los procesos institucionales. (13) Manual de gestión de la seguridad operacional (SMM) Tercera edición 2013 Organización de Aviación Civil Internacional Capítulo 2. Fundamentos de la gestión de la seguridad operacional. (Ver gráfica 3.0)

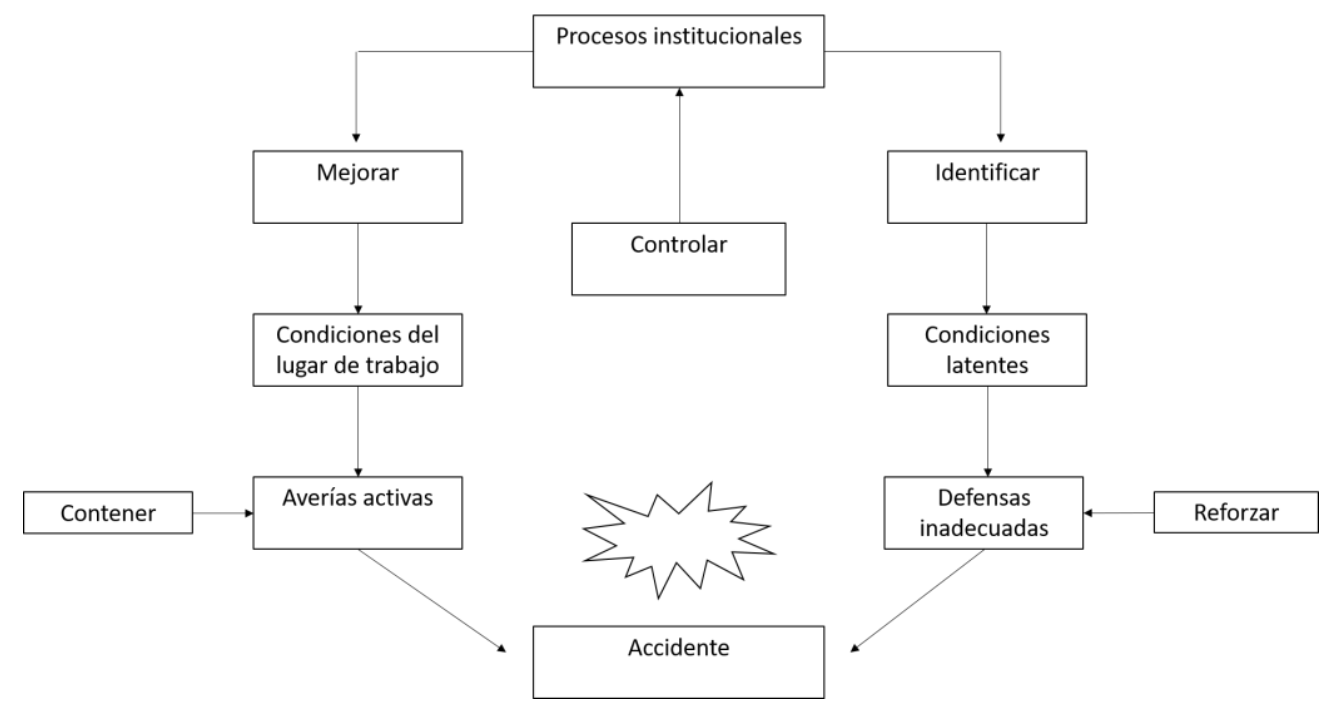

Gráfica 3.0 El accidente institucional

Manual de gestión de la seguridad operacional (SMM) Tercera edición 2013 Organización de Aviación Civil Internacional Capítulo 2. Fundamentos de la gestión de la seguridad operacional. Fig. 2.3

En lo que corresponde a mejorar las condiciones del lugar de trabajo se pretende garantizar que el comportamiento de los individuos sea asertivo como consecuencia de una homeostasis entre su condición mental y psicológica más allá de su comodidad ergonómica, esto conlleva a las organizaciones a mejorar los procesos internos en lo que a la administración del recurso humano se refiere; en lo que corresponde a contener las averías activas se pretende que el comportamiento de los individuos sea direccionado mediante una cultura justa que identifique de manera adecuada la diferencia entre los errores y las violaciones mitigando en ellos la probabilidad de incurrir en errores y evitando las violaciones mediante la aplicación de consecuencias punitivas.

Estos comportamientos no se logran de manera casual ni espontanea, más bien, se debe decir que estos comportamientos son consecuencia o el resultado de las políticas y los procesos organizacionales que identifiquen de manera adecuada a los perfiles requeridos 
para estos estándares, se los entrene y se les evalúe en su posterior desempeño. Se hace necesario que las organizaciones del sector implementen el concepto del accidente institucional como una de las estrategias de administración del recurso humano y a así mismo, promover los comportamientos adecuados

Cuando la norma incluye la necesidad de estructurar a las organizaciones bajo la premisa de las competencias y los comportamientos adecuados y cuándo ésta es interpretada como una herramienta clave en la mitigación del riesgo, se ven resultados reflejados en la casuística de la accidentalidad.

En el caso de la aviación general que representa el porcentaje mayor en la actividad aérea y que se permea casi que exclusivamente bajo la exigencia al cumplimiento de la normativa ya se ven resultados positivos en materia de la probabilidad de los eventos y accidentes como se demuestra en lo presentado por diversos artículos que reúnen la estadística del crecimiento operacional versus el accidente aéreo. (14) "Data Reveal A Continued Downward Trend In Accident Rate" Ver figura (4.)

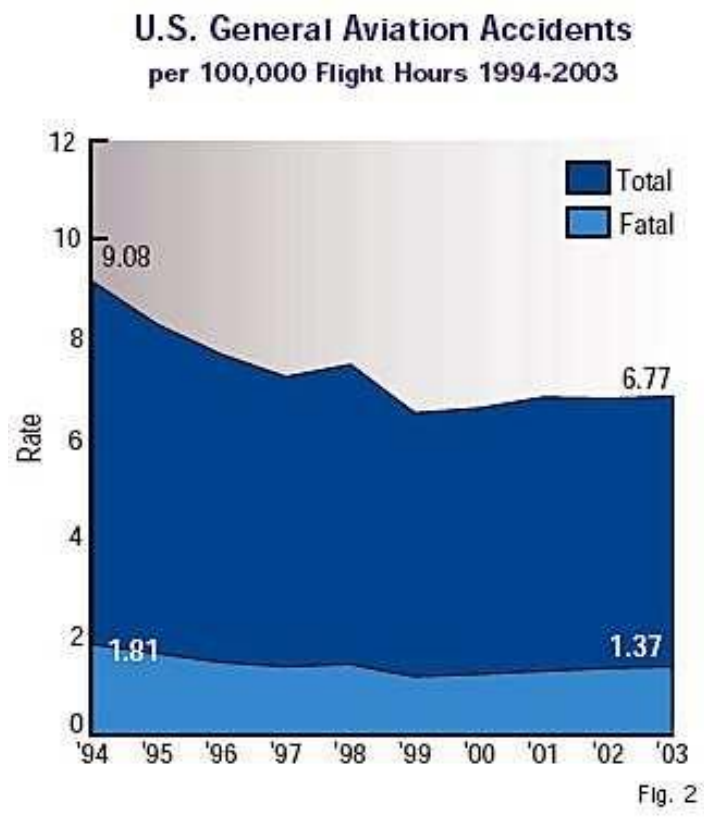

Figura 4.0 Us General Aviation Accidents

http://www.aero-news.net/index.cfm?do=main.textpost\&id=75e59053-2463-4135-b157392320111fab “Data Reveal A Continued Downward Trend In Accident Rate"

Se hace necesario que las autoridades aeronáuticas de los diferentes estados continúen promoviendo la administración del recurso humano basado en la inclusión de las competencias no técnicas y/o actitudinales que han demostrado ser elemento 
diferenciador en los resultados de la accidentalidad, así como, el direccionamiento de los procesos organizacionales o institucionales que orienten la administración del recurso humano en lo ya mencionado, ya que, si bien es cierto los resultados son visibles en cuanto a la probabilidad de manera global, aun en regiones como Latino América menos del diez por ciento de las organizaciones de aviación general cuentan con una estructura organizacional basada en competencias y en sistemas de gestión de la calidad.

\section{Formación de personal aeronáutico}

Usando como referencia a la definición de los conceptos educativos o que hacen parte del sistema de aprendizaje y que describen a las funciones de: Capacitar, formar y entrenar, (15) podemos encontrar:

"Formación: Formación es el desarrollo intelectual o nivel de conocimientos que una persona posee sobre una determinada materia, la formación se equipara a la educación, es un proceso formal, de larga duración y que tiene diversos niveles. La formación no necesariamente se aplica de forma inmediata a lo laboral. La formación es más amplia y contiene dentro de sí a la capacitación y al entrenamiento.

La formación es la primera etapa de desarrollo de un individuo o grupo de individuos que se caracteriza por una programación curricular en alguna disciplina y que permite a quien la obtiene alcanzar niveles educativos cada vez más elevados.

Capacitación: Preparación de una persona para dotarla de conocimientos para ejecutar y desarrollar tareas dentro del ámbito laboral específicos. Por ejemplo, dotar de mayores conocimientos a un empleado.

Entrenamiento: Es de corto plazo. Orientado usualmente a que las personas desarrollen destrezas y habilidades en un determinado puesto de trabajo.

Entre ambos conceptos capacitar y entrenar existe una leve diferencia, la capacitación se centra en dotar de conocimientos y el entrenamiento en desarrollar de habilidades. Sin embargo, en la literatura a veces se emplea entrenamiento y capacitación como sinónimos e incluso no los diferencian."

Éstas definiciones del proceso de aprendizaje plantean un vacío en lo específico dentro del objetivo de promover competencias no técnicas en los individuos que como ya hemos visto hoy por hoy son el pilar de la gestión del riesgo.

En el artículo "Cuatro reflexiones sobre la docencia" publicado por Science Direct, publicado en noviembre de 2017 (16), podemos leer lo siguiente: 
"Dentro de los diferentes elementos que se debe tener en cuenta en la actualidad, se encuentra el impacto de las competencias en la relación educación-trabajo-vida, en donde las competencias serían las habilidades y comportamientos efectivos para el logro o desempeño en trabajos exitosos que proyecten calidad de vida con crecimiento auto sostenible y desarrollo más equitativo. Si bien es cierto que la formación por competencias mejora la productividad y competitividad, permite a los trabajadores acceder a empleo, conservarlo y garantizar la calidad de la formación, no es posible asegurar las dos primeras debido al continuo cambio, incertidumbre y adversidad económica, política y social a la que las sociedades contemporáneas se encuentran enfrentadas.

El cambio metodológico implicado en el aprendizaje por competencias no se ha producido adecuadamente debido a procesos internos y externos de cada uno de los centros educativos en donde se ha implementado"

Los centros de instrucción aeronáutica básica no son excepción ya que aún no incluyen en el proceso de aprendizaje a las competencias no técnicas trabajan todavía bajo el esquema de capacitar y entrenar, así entonces, la formación de competencias blandas o soft skills esenciales en la eficiencia del individuo, es un programa que se utiliza en la aviación de línea o aerolíneas en sus propios centros de entrenamiento avanzado, esta situación crea un gran vacío entre las competencias de los alumnos egresados de las escuelas básicas y las necesidades del sector de las aerolíneas.

Los programas de entrenamiento como el EBT (Evidence based training) contienen como estrategia clara para lograr los objetivos propuestos en la asimilación de contenidos a las competencias no técnicas como se puede ver en el manual de entrenamiento de IATA. (17) IATA (International Air Transport Association) Evidence-Based Training Implementation Guide july 2013 Chapter 3.4

\section{COMPONENTS OF COMPETENCIES- COMPONENTES DE LAS COMPETENCIAS}

"The predominant components of all competencies are the behavioral indicators. The behavioral indicators can be seen as the "assets" or "tools" necessary to enable a crew to operate safely, efficiently and effectively in all phases of flight. Since the introduction of human factor concepts, the industry is using various similar models to describe and relate human behavior with performance."

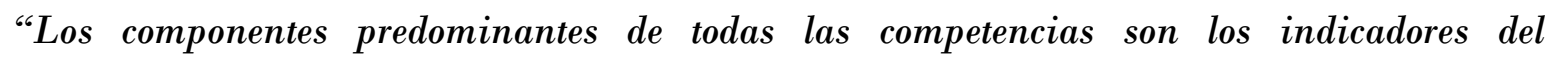
comportamiento. Los indicadores del comportamiento pueden ser vistos como los activos o herramientas necesarios para capacitar a una tripulación para operar de manera segura, eficiente y efectiva en todas las fases de vuelo. Desde la introducción de los conceptos del factor humano, la industria está usando varios modelos similares para describir y relatar el comportamiento humano con rendimiento." 
La normativa que rige a los centros de instrucción aeronáutica aún no es clara y definitiva en la exigencia de la inclusión de las soft skills o competencias no técnicas como parte de los programas y evaluaciones del rendimiento, nuevamente se hace necesario el estudio y la inclusión articulada de las habilidades, el conocimiento y el comportamiento como una estrategia clave en la formación de personal del sistema aeronáutico no solo a nivel de aerolíneas sino que, debe ser incluido a todo nivel si consideramos que el rendimiento en materia de la gestión compete a todo el sistema.

\section{El principio de la integralidad}

Al comprender la actividad aérea como la interrelación que existe entre las organizaciones aeronáuticas podemos decir que la aviación es un sistema, por ello nos referimos sobre la aviación como el sistema aeronáutico, en éste sistema, los individuos que interactúan desde sus organizaciones afectan de manera directa el desempeño del sistema en materia de la seguridad operacional. Es el actuar como consecuencia de las competencias de los individuos lo que finalmente afecta el rendimiento de la seguridad y la gestión del riesgo.

En el artículo "Modelo de gestión organizacional basado en el logro de objetivos" publicado por Science Direct en marzo 2 de 2015 por Yeison Tamayo Salamanca (18) podemos encontrar una reflexión sobre la gestión y la integración del factor humano así:

\section{"DIMENSIÓN DE DESARROLLO HUMANO:}

Entendida como el constante incremento de la calidad de vida personal y de la población, en términos de procesos de aprendizaje, considerando la influencia del contexto donde se convive, a partir de acceso a salud, educación y empleo, y la interacción social encaminada al desarrollo de la personalidad. Es por esto, que la productividad debe tener en cuenta los elementos asociados al desarrollo humano, a partir de la conciencia de la productividad derivada de un proceso autónomo. Para lo anterior, se consideran una serie de factores que se analizan al momento de la construcción de indicadores, como son:

1.Desarrollo humano social: Desarrollo de habilidades individuales en términos de capacidades y conocimientos para establecer relaciones armónicas en un conjunto social.

2.Desarrollo Humano a partir del contexto: En términos de Interacción permanente con la relación pro activa del entorno socioeconómico y ambiental.

3.Desarrollo humano físico corporal: Medido en términos de habilidades motrices, mentales y orgánicas, para optimizar el desempeño de la labor.

4.Desarrollo humano emocional: Es un despliegue integral de emociones para analizar el accionar cotidiano.

5.Desarrollo humano cognitivo-intelectual: Proceso evolutivo de aprendizaje, con el cual, se potencializa el desarrollo de la conciencia desencadenado en acciones." 
Considerando lo anterior y con el ánimo de proveer a los individuos en las competencias adecuadas para su desempeño, el sistema aeronáutico ha evolucionado en el entendimiento de la gestión del riesgo y con ello la estrategia, hoy, los individuos que cumplen la actividad aérea deben demostrar competencias en tres aspectos clave: habilidad, conocimiento y actitud.

Sin embargo, y como ya lo hemos mencionado, aunque el sistema aeronáutico ha evidenciado de manera clara las necesidades en competencias humanas, la identificación de las mismas en los procesos de selección de personal y en el entrenamiento básico aún no se da de manera general, en las organizaciones del sector aéreo existe aún hoy en día un grado alto de desconocimiento de las competencias no técnicas como elemento base para los procesos de administración y formación del recurso humano.

Éste panorama es consecuencia entre otras cosas debido a las diferencias culturales de región y si bien es cierto que existen las recomendaciones de mejora desde las diferentes organizaciones internacionales, la normativa particular en los diferentes países apenas está incluyendo esta necesidad de manera informativa. Las organizaciones (en su mayoría de aviación general) aún no identifican a las competencias como una necesidad de gestión, abona a este diagnóstico el hecho de que las organizaciones de aviación general no tienen una cultura generalizada sobre los sistemas de aseguramiento a la calidad lo que hace más difícil la comprensión de las necesidades de gestión basadas en el factor humano.

En el artículo "El principio de la integralidad" publicado en octubre de 2020 por el autor: (19) se plantea la integración articulada de las competencias técnicas, cognitivas y actitudinales. "El integrar articuladamente a las competencias técnicas, cognitivas y actitudinales disminuye la probabilidad y la severidad de los eventos en materia de la seguridad".

La integralidad como concepto no es nuevo, lo que sí es nuevo es la inclusión de éste principio en los procesos de selección, formación, promoción y evaluación del personal aeronáutico a todo nivel incluyendo los componentes organizacionales más allá de la línea de vuelo.

Si las organizaciones del sistema aeronáutico promueven la norma de la integralidad en las competencias referidas, se promueve de manera clara la necesidad de la inclusión de las competencias no técnicas en la administración del recurso humano, una vez más se propondría y regularía de manera precisa la inclusión de los programas especiales de gestión del riesgo a todo nivel.

El profesor Fabio Losada en su artículo "La integralidad del desarrollo humano a través de los proyectos educativos institucionales" (20), publicado en Revistas científicas editorial USCO para la universidad Surcolombiana expone: "La base fundamental de la integralidad de los currículos y a la vez que respondan a las necesidades del medio debe estar basada en los ejes fundamentales y prácticos del desarrollo humano" 
Por lo anterior y como esta mencionado en el principio de la integralidad, el entrenamiento del personal y la evaluación contrastada contra los estándares actuales, deberá incluir la participación de los profesionales en psicología aeronáutica mucho más allá del aspecto clínico para convertirlos en formadores y promotores de la integralidad en competencias.

Como se ha visto, la gestión del riesgo ha requerido competencias en el personal aeronáutico que hoy en día se integran de manera articulada, según el autor el principio de la integralidad ésta se define como: "La convergencia obligada que deben demostrar los individuos de la industria en tres aspectos claves del desempeño: Las habilidades técnicas, las habilidades cognitivas y las habilidades actitudinales". (Ver gráfica 3.0).

\section{PRINCIPIO DE LA INTEGRALIDAD}

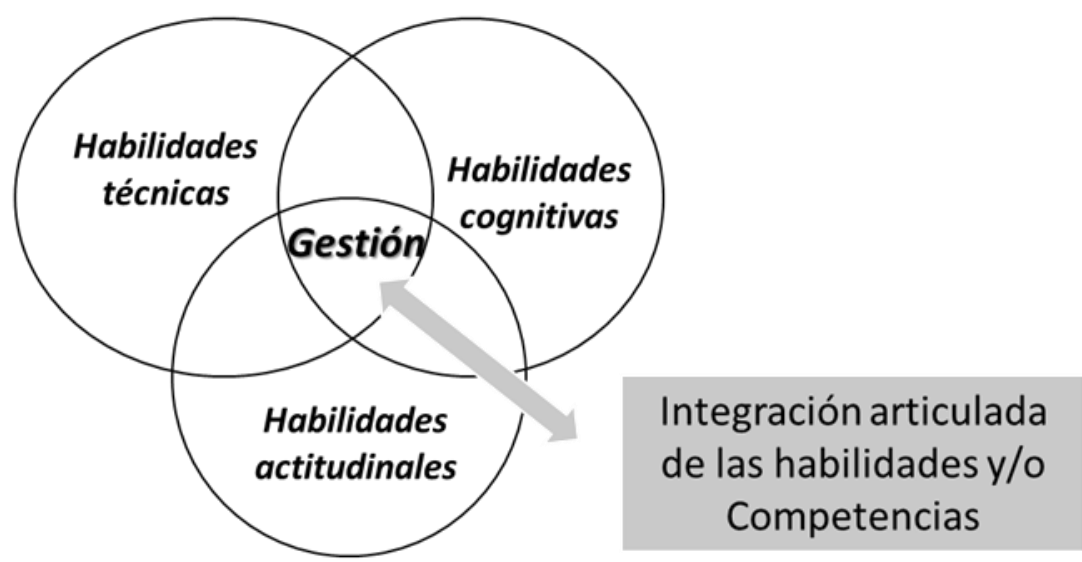

Gráfica 3.0

El principio de la integralidad

https:/osf.io/preprints/socarxiv/r9gze/

Si bien es cierto que las competencias técnicas y cognitivas son indispensables en la gestión del riesgo, son las competencias actitudinales o no técnicas las que han demostrado definitivamente tener un efecto sobre la probabilidad y la severidad de los eventos materia de la accidentalidad.

\section{Conclusiones}

La seguridad operacional como cultura es la evolución del pensamiento en materia de la gestión de la seguridad, esta cultura promueve las políticas de mitigación del riesgo a todo nivel organizacional, es indispensable que los ejecutivos responsables de las diferentes organizaciones evidencien la necesidad de incluir la integralidad de las competencias en los 
individuos ya que, es allí en donde se ha evidenciado un real avance sobre la disminución de la probabilidad de los accidentes.

Al considerar a la aviación como un sistema en el cual interactúan las diferentes organizaciones (Autoridades aeronáuticas, centros de instrucción, operadores, ATC, talleres, etc.) es necesario entender que la interrelación de las organizaciones en éste sistema se da por el comportamiento de los individuos que las integran y que, sin lugar a duda éste comportamiento afecta al sistema de manera general, por lo tanto identificar, formar y evaluar las competencias de los individuos es esencial en la afectación del resultado de la operación aérea, los procesos de selección y entrenamiento basados en evidencias y competencias son la clave en la implementación de la cultura organizacional.

Teniendo en cuenta las estrategias desarrolladas desde las diferentes organizaciones internacionales quienes de acuerdo a la casuística generaron los programas especiales de gestión del riesgo y una vez más, identificando la necesidad de integrar las competencias no técnicas a lo ya realizado en el compendio de la habilidad y el conocimiento, debemos propender por la regulación de éstas mediante la normativa desde la autoridad aeronáutica, ya no de manera informativa sino que, más bien, promoviendo su aplicación y evaluación mediante los informes de rendimiento del sistema de gestión de la seguridad operacional.

Los centros de instrucción aeronáutica deberán incluir el principio de la integralidad como la herramienta que les permita generar perfiles en los individuos que aspiran a desempeñarse en el sistema, siendo los centros de instrucción al mismo tiempo operadores de aeronaves y formadores de personal se deberán incluir las competencias de manera integrada a nivel de instructores, docentes y alumnos, actualizando para ello los programas académicos y de entrenamiento bajo los lineamientos de la integralidad en competencias, considerando además que el profesional en psicología aeronáutica deberá ser parte activa del proceso de formación y evaluación de los alumnos como promotor de las competencias blandas o soft skills.

Con lo anterior, el sistema aeronáutico de manera general debe optimizar el proceso de selección, formación, promoción y evaluación del personal, ejecutándose mediante el principio de la integralidad con el objetivo de garantizar las competencias necesarias a todo nivel organizacional como lo establece la cultura misma de la seguridad operacional.

Se recomienda finalmente el desarrollo de un estudio desde las asociaciones de psicología aeronáutica y los centros de instrucción aeronáutica correlacionados con la operación aérea, en el que se evidencie cómo la participación del profesional en psicología aeronáutica durante el desarrollo los procesos de entrenamiento y evaluación de personal, así como, en el desarrollo de los programas especiales de gestión del riesgo, mejora el 
rendimiento del individuo en las competencias no técnicas y en el desempeño final durante la actividad aérea.

\section{Referencias:}

(1) OACI Documento 9859 Capítulo 2, parte 2.2.4 Significado de la seguridad operacional

(2) "La conducta humana frente a los riesgos laborales: determinantes individuales y grupales" https://doi.org/10.26754/ojs ais/ais.200112199

(3) OACI Documento 9859 Capítulo 2, parte 2.8 Cultura de organización (corporativa)

(4) Boletín de la Organización de Aviación Civil Internacional, volumen 61, núm. 6, Pag.

(5) Reglamento Aeronáutico de Colombia parte 219.105

(6) Andrew R. Hale \& Jan Jovden en el texto Ocupational injury, risk prevention and intervention, capítulo 11, Management and culture, the third age of safety, a review of approaches to organizational aspects of safety, healt and enviroment. 1998

(7) OACI Doc. 9683 "Manual de. Instrucción sobre Factores Humanos"

(8) https://www.skybrary.aero/index.php/Evidence based training_(EBT)

(9) El principio de la integralidad 2020 https://osf.io/preprints/socarxiv/r9gze/

(10) $\quad$ https://www.easa.europa.eu/sites/default/files/dfu/HE8 ES.pdf

(11) https://safetyfirst.airbus.com/learning-from-the-evidence/

(12) La cultura organizacional, su importancia en el desarrollo de las empresas https://doi.org/10.33890/innova.v2.n3.2017.188

(13) Manual de gestión de la seguridad operacional (SMM) Tercera edición 2013 Organización de Aviación Civil Internacional Capítulo 2. Fundamentos de la gestión de la seguridad operacional.

(14) $\quad$ http://www.aero-news.net/index.cfm?do=main.textpost\&id=75e590532463-4135-b157-392320111fab, “ Data Reveal A Continued Downward Trend In Accident Rate"

(16) Cuatro reflexiones sobre la docencia"

https://doi.org/10.1016/j.reper.2017.09.001

(17) IATA (International Air Transport Association) Evidence-Based

Training Implementation Guide july 2013 chapter 3.4

(18) "Modelo de gestión organizacional basado en el logro de objetivos https://oi.org/10.1016/S2215-910X(14)70021-7

(19) Bustamante, R. D. L. (2020, October 27). El Principio de la Integralidad. https://doi.org/10.31235/osf.io/r9gze

(20) La integralidad del desarrollo humano a través de los proyectos educativos institucionales https://doi.org/10.25054/01240307.929 


\section{Palabras clave:}

Aviación; Factores humanos; Gestión del riesgo; Integralidad; seguridad operacional, psicología aeronáutica.

\begin{tabular}{|l|l|}
\hline \multicolumn{2}{|c|}{ DATOS DEL AUTOR } \\
\hline Nombres & Rubén Darío \\
\hline Apellidos & Lésmes Bustamante \\
\hline ORCID ID & $0000-0003-3669-2208$ \\
\hline Currículum & $\begin{array}{l}\text { Piloto instructor, docente y conferencista internacional, } \\
\text { consultor latinoamericano del sistema aeronáutico, } \\
\text { especialista en seguridad operacional y factores humanos, } \\
\text { escritor del principio de la integralidad. }\end{array}$ \\
\hline $\begin{array}{l}\text { Función dentro del } \\
\text { artículo }\end{array}$ & \begin{tabular}{l} 
Escritor \\
\hline Dirección electrónica
\end{tabular} \\
\hline Dirección postal & $\begin{array}{l}\text { Calle 14 \# 3-2 torre 3 Apto. (411) Portal del café, San Gil } \\
\text { (Santander.) }\end{array}$ \\
\hline Teléfono de contacto & +573505820640 \\
\hline
\end{tabular}

